\title{
Analytical carbon-oxygen reactive potential
}

\author{
A. Kutana and K. P. Giapis ${ }^{\text {a) }}$ \\ Division of Chemistry and Chemical Engineering, California Institute of Technology, Pasadena, \\ California 91125, USA
}

(Received 22 January 2008; accepted 9 May 2008; published online 19 June 2008)

\begin{abstract}
We present a reactive empirical potential with environment-dependent bond strengths for the carbon-oxygen $(\mathrm{CO})$ system. The distinct feature of the potential is the use of three adjustable parameters characterizing the bond: the strength, length, and force constant, rather than a single bond order parameter, as often employed in these types of potentials. The values of the parameters are calculated by fitting results obtained from density functional theory. The potential is tested in a simulation of oxidative unzipping of graphene sheets and carbon nanotubes. Previous higher-level theoretical predictions of graphene unzipping by adsorbed oxygen atoms are confirmed. Moreover, nanotubes with externally placed oxygen atoms are found to unzip much faster than flat graphene sheets. (C) 2008 American Institute of Physics. [DOI: 10.1063/1.2940329]
\end{abstract}

\section{INTRODUCTION}

Empirical interatomic potentials offer substantial advantages in calculating the properties of large statistical entities as compared to more rigorous $a b$ initio or tight binding methods, which typically produce more accurate results albeit for much smaller systems. In particular, reactive empirical bond order (REBO) potentials have been very successful in simulating covalently bonded materials such as Si (Ref. 1) and hydrocarbons. ${ }^{2,3}$ They have also facilitated the description of chemical reactions and barriers in large systems ${ }^{4}$ and established molecular dynamics simulations as an important tool in designing new materials, estimating their properties, and understanding materials processing. This success motivated the development of REBO potentials for chemically complex systems of different atoms. ${ }^{5-7}$

The REBO approach is based on a fixed relationship between bond energy and bond order, which is a function of the coordination numbers of atom pairs forming a bond. This empirical relationship is only approximately satisfied in real molecules and may yield imprecise results especially in systems encompassing two or more chemically distinct atoms. Each new atom considered introduces a new set of parameters, which must be optimized by fitting the equilibrium distance and energy of bonds formed between the new atom and the previous set of atoms. This fitting strategy is not specific to the REBO potential. It can be implemented to other potentials with their own sets of parameters, provided they are complex enough to capture an increasing number of bonding configurations.

We present here a new approach to generating empirical reactive potentials for covalent systems. We focus on the carbon-oxygen system as a model. Carbon-oxygen chemical reactions are ubiquitous, from combustion to metabolic reactions in cells. ${ }^{8}$ The importance of carbon-oxygen chemistry

\footnotetext{
a) Author to whom correspondence should be addressed. Electronic mail: giapis@cheme.caltech.edu.
}

has led to many electronic structure and potential energy surface calculations for oxygen-hydrocarbon interactions using $a b$ initio methods. ${ }^{9-12}$ Yet, fast analytical interatomic potentials capable of capturing the chemical interactions between these elements have been scarce. In fact, there exists currently only one such potential ${ }^{6}$ to our best knowledge. In order to fill this gap, a carbon-oxygen potential based on an extensive fitting database of $a b$ initio calculations is presented here. The motivation for this work has also been an attempt to avoid any predefined fixed relationships between bond energy, length, and force constants present in traditional bond order potentials.

In Sec. II, we describe the formalism used to represent the binding energy as a function of the environment of a chemical bond. In Sec. III, the parameters of the formulas given in Sec. II are fitted to results of $a b$ initio calculations. In Sec. IV, the $\mathrm{C}-\mathrm{O}$ potential developed is applied to the problem of unzipping of graphene sheets and carbon nanotubes by adsorbed oxygen. Conclusions are given in Sec. V.

\section{REACTIVE POTENTIAL}

One of the most successful approaches to modeling reactive chemistry in atomistic simulations has been the bond order method. ${ }^{1}$ It introduces environment-dependent corrections to the strengths of chemical bonds based on the local coordination of atoms. Unlike multibody potential expansions, where each successive term depends on one extra atomic coordinate, the bond order formalism retains the form of a pair potential; instead, all the corrections are included into the coefficients of the potential function. Previous reactive potentials for silicon ${ }^{1}$ and hydrocarbons $(\mathrm{C}+\mathrm{H} \text { system })^{2}$ used a bond order parameter $b_{i j}\left(b_{i j} \leqslant 1\right)$ to account for the environment-dependent bond order between atoms $i$ and $j$ as follows: 


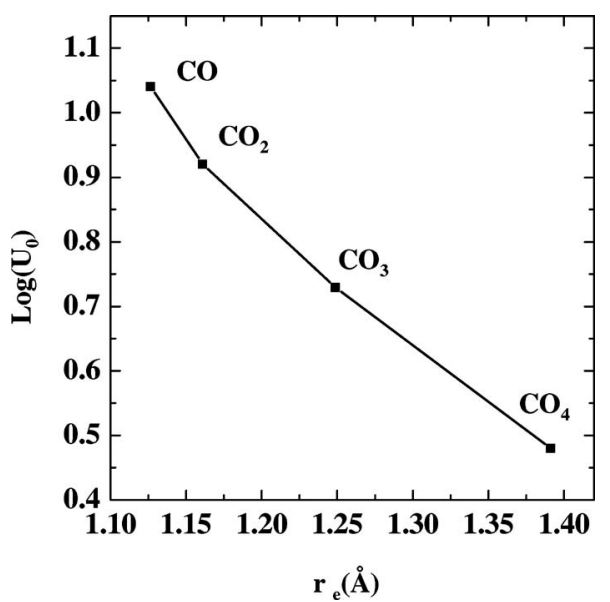

FIG. 1. The Pauling plot for the potential well depth and equilibrium bond length of the $\mathrm{CO}$ bond represented by a Morse potential with variable coefficients as a function of the number of the $\mathrm{O}$ atoms connected to a $\mathrm{C}$ atom. The molecular energies are obtained using GAMESS (DFT, 6-311G*, and B3LYP density functional).

$$
U_{i j}=f_{c}\left(r_{i j}\right)\left(V_{R}\left(r_{i j}\right)-b_{i j} V_{A}\left(r_{i j}\right)\right) .
$$

Here, $r_{i j}$ is the distance between atoms $i$ and $j, f_{c}\left(r_{i j}\right)$ is the cutoff function used for smooth potential truncation, and $V_{R}\left(r_{i j}\right)$ and $V_{A}\left(r_{i j}\right)$ are the repulsive and attractive interactions usually given by Morse-type expressions

$$
\begin{aligned}
& V_{R}(r)=A \exp \left(-\alpha_{1} r\right), \\
& V_{A}(r)=B \exp \left(-\alpha_{2} r\right),
\end{aligned}
$$

where $A, B, \alpha_{1}$, and $\alpha_{2}$ are all positive $\left(\alpha_{1}=2 \alpha_{2}=\alpha\right.$ in the original Morse potential). The total bonding energy is obtained by summing $U_{i j}$ over all bonds as follows:

$$
E_{\mathrm{bond}}=\sum_{j>i} U_{i j} \text {. }
$$

In a more recent REBO potential for hydrocarbons, ${ }^{3}$ a repulsive term of the form

$$
V_{R}(r)=(1+Q / r) A \exp \left(-\alpha_{1} r\right)
$$

was introduced, where $Q$ is a fitting parameter. The modified pre-exponential factor $1+Q / r$ accounts for Coulombic repulsion, thus rectifying the unphysical behavior of the Morse potential at small $r$.

Morse-type functions are well suited for capturing the universal energetics of covalent and metallic bonds. ${ }^{13,14}$ The bond order parameter $b_{i j}$ depends on the atomic coordination in a molecule or solid and is central to the potential's ability to reproduce correct binding energies. The form of Eqs. (1) and (2) (with $\alpha_{1}=2 \alpha_{2}=\alpha$ ) requires that bond energies and bond distances follow the Pauling rule, ${ }^{15}$ stating that the logarithm of the bond order (bond strength) $U_{0}$ is a linear function of the equilibrium bond distance $r_{e}$ as follows:

$$
\log U_{0}=\log A-2 \alpha r_{e} .
$$

Figure 1 shows the energies of $\mathrm{CO}_{n} n=1,4$ molecules calculated using density functional theory (DFT). The Pauling rule is followed only approximately: the $\mathrm{CO}_{3}$ and $\mathrm{CO}_{4}$ molecules are more stable than what would follow from a linear fit for the $\mathrm{CO}$ and $\mathrm{CO}_{2}$ molecules. Usually, a best fit to the Pauling rule is performed in order to minimize the errors over different coordinations. The fitting could also be improved by introducing corrections to the angular energy of the molecule, at the cost of a more complex expression for it. As an alternative to these approaches, we remove the requirement that the approximate Pauling rule be satisfied, and allow all three parameters $A, B$, and $\alpha$ in Eqs. (1) and (2) to vary independently. Using the parameters

$U_{0}=B^{2} / 4 A$ and $r_{e}=(1 / \alpha) \ln (2 A / B)$, which are the potential well depth and equilibrium interatomic distance, respectively, instead of $A$ and $B$ in Eq. (2), the expression for the bond energy becomes

$$
U_{i j}=f_{c}\left(r_{i j}\right) U_{0}\left(\exp \left(-2 \alpha\left(r_{i j}-r_{e}\right)\right)-2 \exp \left(-\alpha\left(r_{i j}-r_{e}\right)\right)\right),
$$

where $U_{0}, r_{e}$, and $\alpha$ are functions of the numbers of neighbors on both sides of the bond between atoms $i$ and $j$ as follows:

$$
\begin{aligned}
& U_{0}=U_{0}\left(N_{\mathrm{C} i}, N_{\mathrm{O} i}, N_{\mathrm{C} j}, N_{\mathrm{O} j}\right), \\
& r_{e}=r_{e}\left(N_{\mathrm{C} i}, N_{\mathrm{O} i}, N_{\mathrm{C} j}, N_{\mathrm{O} j}\right), \\
& \alpha=\alpha\left(N_{\mathrm{C} i}, N_{\mathrm{O} i}, N_{\mathrm{C} j}, N_{\mathrm{O} j}\right) .
\end{aligned}
$$

Here, $N_{\mathrm{C} i}$ is the number of carbon atoms connected to atom $i$ of the $i j$ bond, $N_{\mathrm{O} i}$ is the number of oxygen atoms connected to this atom, while $N_{\mathrm{C} j}$ and $N_{\mathrm{O} j}$ are similar quantities for atom $j$. Each parameter is a function of $2 K$ variables, where $K$ is the total number of the atomic species in the system. The coefficients $U_{0}, r_{e}$, and $\alpha$ are calculated for stable molecules where bonds are assigned integers $N_{\mathrm{C}, \mathrm{O} i}$ and $N_{\mathrm{C}, \mathrm{O} j}$. Use of fractional $N$ values permits smooth switching through different coordinations and allows chemical reactions to occur.

The particular form of the smooth interpolating functions used here for fitting the parameters in Eq. (7) as functions of the number of neighbors is a multivariate polynomial of $m$ variables of the order $n$ as follows:

$P_{n}\left(x_{1}, x_{2}, \ldots, x_{m}\right)=\sum_{i=0}^{n} x_{1}^{i} \sum_{j=0}^{n-i} x_{2}^{j} \sum_{k=0}^{n-i-j} x_{3}^{k} \ldots \sum_{l=0}^{n-i-j-k-\ldots} a_{i j k \ldots l} x_{m}^{l}$,

where $x_{i}$ are the variables corresponding to $N_{\mathrm{C} i}, \ldots, N_{\mathrm{O} j}$ in Eq. (7), and $a_{i j k \cdots l}$ are the coefficients of the polynomial. The 
number of polynomial variables $m$ is equal to twice the number of the present reactive species, which is 2 for the current $\mathrm{CO}$ system, hence, $m=4$. The degree $n$ of the polynomial is $n=3$. The 35 unknown coefficients $a_{i j k l}$ in $P_{3}$ are found by solving a linear system of equations as follows:

$$
P_{3}(q)=\sum_{i=0}^{3} x_{1}^{i}(q) \sum_{j=0}^{n-i} x_{2}^{j}(q) \sum_{k=0}^{n-i-j} x_{3}^{k}(q) \sum_{l=0}^{n-i-j-k} a_{i j k l} x_{4}^{l}(q),
$$

where $q=1,2, \ldots, 35$ enumerates points in the fourdimensional fitting space. The numbers of neighbors $x_{1}, \ldots, x_{4}$ on each side of the bond are calculated according to

$$
\begin{aligned}
& x_{1} \equiv N_{\mathrm{C} i}=\sum_{k \in \mathrm{C} \text { near } i} f_{c}\left(r_{i k}\right)\left(1-f_{c}\left(r_{j k}\right)\right), \\
& x_{2} \equiv N_{\mathrm{O} i}=\sum_{k \in \mathrm{O} \text { near } i} f_{c}\left(r_{i k}\right)\left(1-f_{c}\left(r_{j k}\right)\right), \\
& x_{3} \equiv N_{\mathrm{C} j}=\sum_{k \in \mathrm{C} \text { near } j} f_{c}\left(r_{j k}\right)\left(1-f_{c}\left(r_{i k}\right)\right), \\
& x_{4} \equiv N_{\mathrm{O} j}=\sum_{k \in \mathrm{O} \text { near } j} f_{c}\left(r_{j k}\right)\left(1-f_{c}\left(r_{i k}\right)\right),
\end{aligned}
$$

where $f_{c}\left(r_{i k}\right)$ is the cutoff function

$$
f_{c}(r)= \begin{cases}1, & r \leqslant D_{\min } \\ {\left[1+\cos \left(\pi\left(r-D_{\min }\right) /\left(D_{\max }-D_{\min }\right)\right)\right] / 2,} & D_{\min }<r<D_{\max } \\ 0, & r \geqslant D_{\max }\end{cases}
$$

for which the appropriate parameters $D_{\max }$ and $D_{\min }$ are taken for an atomic pair $i k$. The numerical values of $D_{\max }$ and $D_{\min }$ for different atomic pairs are listed in Table I. Note that according to Eq. (10), an atom, which is connected to both atoms constituting the bond, is not considered to be a neighbor of either of these atoms.

In addition to the bonding energy, the full form of the total potential energy also includes the angular, dihedral, and van der Walls (vdW) components as follows:

$$
\begin{aligned}
E_{\mathrm{total}} & =E_{\mathrm{bond}}+E_{\mathrm{angle}}+E_{\mathrm{tor}}+E^{\mathrm{vdW}} \\
& =\sum_{j>i} U_{i j}+\sum_{j, i, k} E_{j i k}^{\mathrm{angle}}+\sum_{k, i, j, l} E_{k i j l}^{\mathrm{tor}}+E^{\mathrm{vdW}} .
\end{aligned}
$$

The three-atom angular energy $E_{j i k}^{\text {angle }}$ is presented by

$$
E_{j i k}^{\text {angle }}\left(\theta_{j i k}\right)=f_{c}\left(r_{i j}\right) f_{c}\left(r_{i k}\right) \frac{1}{2} k^{\text {angle }}\left(\theta_{j i k}-\theta_{0}\right)^{2},
$$

where $\theta_{j i k}$ is the angle formed by the adjacent bonds $i j$ and $i k$, and $\theta_{0}$ is the equilibrium angle. The cutoff function factors ensure smooth truncation of the angular term upon dissociation of either of the bonds in the angle. The dihedral, or torsional energy $E_{k i j l}^{\text {tor }}$ is described as

$$
E_{k i j l}^{\mathrm{tor}}\left(\phi_{k i j l}\right)=f_{c}\left(r_{i j}\right) f_{c}\left(r_{i k}\right) f_{c}\left(r_{j l}\right) \frac{1}{2} k^{\mathrm{tor}}\left(1-\cos ^{2} \phi_{k i j l}\right),
$$

where $\phi_{k i j l}$ is the torsional angle between the two planes formed by triplets of atoms $k i j$ and $i j l$ centered on the $i j$ bond. Again, the cutoff functions are added for eliminating

TABLE I. Values of the parameters $D_{\min }$ and $D_{\max }$ used in the cutoff function.

\begin{tabular}{ccc}
\hline \hline & $D_{\min }(\AA)$ & $D_{\max }(\AA)$ \\
\hline $\mathrm{C}-\mathrm{C}$ & 1.7 & 2.0 \\
$\mathrm{C}-\mathrm{O}$ & 1.7 & 2.0 \\
$\mathrm{O}-\mathrm{O}$ & 1.5 & 1.9 \\
\hline \hline
\end{tabular}

the dihedral term when one or more of the bonds constituting the angle dissolve. In the current version of the potential, the spring constants and equilibrium angle in Eqs. (13) and (14) are insensitive to the local environment. Since the equilibrium angle is fixed at $180^{\circ}$ in most cases, the angular components in nonlinear molecules at equilibrium may not be zero and will contribute to the total energy of these molecules. As a result, the bonding and angular energies are coupled via the total energy. The coupling mandates that bonding and angular terms be fitted simultaneously to yield the correct total energy of the molecule.

At very short interatomic distances, the Morse-type potential (1) approaches a finite value, while in reality the interaction follows the Coulombic repulsion with $1 / r$ scaling. For a more realistic description of the interaction at short $r$, a screened Coulomb potential function has been introduced into the total potential function in that region. A two-body Moliere potential ${ }^{16}$

$$
\begin{aligned}
U_{M}(r)= & (A / r)\left(0.35 \exp \left(-0.3 r / a_{s}\right)+0.55 \exp \left(-1.2 r / a_{s}\right)\right. \\
& \left.+0.1 \exp \left(-6.0 r / a_{s}\right)\right)
\end{aligned}
$$

represents the interaction between a pair of atoms as given by the Thomas-Fermi model. Here, $A / r$ is the Coulomb

TABLE II. Parameters of the angular function for the CO potential.

\begin{tabular}{ccc}
\hline \hline Angle & $\theta_{0}(\mathrm{deg})$ & $k_{\text {ang }}(\mathrm{eV})$ \\
\hline $\mathrm{O}-\mathrm{C}-\mathrm{O}$ & 180.0 & 4.56 \\
$\mathrm{O}-\mathrm{C}-\mathrm{C}$ & 180.0 & 1.85 \\
$\mathrm{C}-\mathrm{O}-\mathrm{C}$ & 180.0 & 1.89 \\
$\mathrm{C}-\mathrm{C}-\mathrm{C}$ & 180.0 & 3.04 \\
$\mathrm{O}-\mathrm{O}-\mathrm{O}$ & 118.2 & 14.0 \\
$\mathrm{O}-\mathrm{O}-\mathrm{C}$ & 180.0 & 1.37 \\
\hline
\end{tabular}


TABLE III. Calculated parameters of the molecules used in the fitting database. Molecules are in a singlet state, unless indicated otherwise. Bond types in column 3 are labeled by the symbols of the elements forming the bond, i.e., $\mathrm{CO}$, followed by numbers in parentheses signifying how many neighbors of each atomic species each side of the bond has. For instance, $\mathrm{CO}(2110)$ designates a carbon-oxygen bond with two carbons and one oxygen connected to a carbon atom and one carbon and no oxygen connected to an oxygen atom.

\begin{tabular}{|c|c|c|c|c|c|}
\hline Molecule & Atomization $E(\mathrm{eV})$ & Bond & Bond order & $r_{e}(\AA)$ & $k\left(\mathrm{eV} / \AA^{2}\right)$ \\
\hline $\mathrm{O}-\mathrm{C}$ (carbon monoxide) & -10.9787 & $\mathrm{CO}(0000)$ & 2.408 & 1.1265 & 123.4 \\
\hline $\mathrm{O}-\mathrm{C}-\mathrm{O}$ (carbon dioxide) & -16.6458 & $\mathrm{CO}(0100)$ & 2.097 & 1.1609 & 102.0 \\
\hline $\mathrm{O}-\mathrm{CO}_{2}$ & -16.0837 & $\mathrm{CO}(0200)$ & 1.521 & 1.2487 & 60.4 \\
\hline $\mathrm{O}-\mathrm{CO}_{3}$ (triplet) $T_{d}$ & -12.0881 & $\mathrm{CO}(0300)$ & 1.085 & 1.3913 & 12.7 \\
\hline $\mathrm{C}_{2} \mathrm{O}$ (triplet) $D_{\infty h}$ & -10.8373 & $\mathrm{CO}(0010)$ & 1.259 & 1.2586 & 34.0 \\
\hline $\mathrm{C}_{3} \mathrm{O} \mathrm{D}_{3 h}$ & -5.72722 & $\mathrm{CO}(0020)$ & 0.802 & 1.4683 & 1.3 \\
\hline \multirow[t]{2}{*}{$\mathrm{C}-\mathrm{O}-\mathrm{O} *$ (singlet) $C_{\infty v}$} & -9.33251 & $\mathrm{CO}(0001)$ & 1.992 & 1.1540 & 96.5 \\
\hline & & $\mathrm{OO}(1000)$ & 0.779 & 1.3592 & 17.8 \\
\hline \multirow{2}{*}{$\mathrm{C}-\mathrm{O}_{3}$} & -7.33092 & $\mathrm{OO}(1100)$ & 0.725 & 1.4118 & 18.0 \\
\hline & & $\mathrm{CO}(0002)$ & 1.423 & 1.2280 & 59.9 \\
\hline \multirow{2}{*}{$\mathrm{O}-\mathrm{C}-\mathrm{C}-\mathrm{O}$ (triplet) $D_{\infty h}$} & -20.2584 & $\mathrm{CC}(0101)$ & 1.611 & 1.2806 & 72.5 \\
\hline & & $\mathrm{CO}(1000)$ & 2.075 & 1.1871 & 86.4 \\
\hline \multirow[t]{2}{*}{$\mathrm{C}_{2} \mathrm{O}_{2} C_{2 v}$} & -16.0033 & $\mathrm{CC}(0002)$ & 1.011 & 1.4208 & 32.4 \\
\hline & & $\mathrm{CO}(1100)$ & 1.645 & 1.2452 & 35.5 \\
\hline \multirow[t]{2}{*}{$\mathrm{O}-\mathrm{C}-\mathrm{C}-\mathrm{C} C_{\infty h}$} & -21.5027 & $\mathrm{CC}(0110)$ & 1.448 & 1.2952 & 66.3 \\
\hline & & $\mathrm{CO}(1000)$ & 2.247 & 1.1497 & 108.3 \\
\hline \multirow[t]{2}{*}{$\mathrm{O}-\mathrm{C}-\mathrm{C}$ (triplet) $C_{\infty}$} & -13.6562 & $\mathrm{CO}(1000)$ & 2.162 & 1.1627 & 96.3 \\
\hline & & $\mathrm{CC}(0100)$ & 1.595 & 1.3595 & 40.7 \\
\hline \multirow[t]{2}{*}{$\mathrm{C}_{4} \mathrm{O}$ (triplet) $C_{3 v}$ all $\mathrm{C}$ atoms in one plane } & -12.9775 & $\mathrm{CC}(0021)$ & 0.948 & 1.4385 & 23.9 \\
\hline & & $\mathrm{CO}(3000)$ & 0.757 & 1.4184 & 33.9 \\
\hline $\mathrm{C}-\mathrm{C}-\mathrm{O}-\mathrm{C}(\text { singlet })^{\mathrm{a}}$ & -16.8462 & $\mathrm{CO}(1010)$ & 0.903 & 1.3029 & 41.4 \\
\hline $\mathrm{C}-\mathrm{C}$ (dicarbon) & -5.08928 & $\mathrm{CC}(0000)$ & 3.470 & 1.2514 & 77.2 \\
\hline $\mathrm{C}-\mathrm{C}-\mathrm{C}$ (linear tricarbon) & -13.7118 & $\mathrm{CC}(1000)$ & 1.715 & 1.2915 & 67.5 \\
\hline $\mathrm{O}-\mathrm{C}-\mathrm{C}-\mathrm{O}$ (triplet) & -20.2584 & $\mathrm{CC}(0101)$ & 1.611 & 1.281 & 72.5 \\
\hline$C_{4}$ (triplet) $D_{3 h}$ & -14.1619 & $\mathrm{CC}(2000)$ & 1.152 & 1.4103 & 28.0 \\
\hline $\mathrm{C}_{5} D_{4 h}^{\mathrm{b}}$ (triplet) & -18.0646 & $\mathrm{CC}(3000)$ & 0.854 & 1.4109 & 26.4 \\
\hline $\mathrm{C}_{4}$ linear & -17.9933 & $\mathrm{CC}(1010)$ & 1.845 & 1.2961 & 63.4 \\
\hline $\mathrm{O}-\mathrm{O}$ (triplet) & -5.2686 & $\mathrm{OO}(0000)$ & 1.748 & 1.2065 & 79.1 \\
\hline $\mathrm{O}_{3}$ (ozone, singlet) $C_{2 v}$ & -5.8110 & $\mathrm{OO}(0100)$ & 1.248 & 1.2584 & 45.8 \\
\hline graphene $^{c}$ & -7.9173 & $\mathrm{CC}(2020)$ & 1.303 & 1.4189 & 45.0 \\
\hline diamond ${ }^{c}$ & -7.7737 & $\mathrm{CC}(3030)$ & 1.000 & 1.5417 & 24.0 \\
\hline
\end{tabular}

${ }^{\mathrm{a}}$ Triplet calculation diverges.

${ }^{\mathrm{b}}$ Singlet state diverges; pentet state is higher in energy.

${ }^{c}$ Energy per atom.

repulsion between a pair of bare nuclei, while $a_{s}$ is the Firsov ${ }^{17}$ screening length

$$
a_{s}=0.88534 a_{\mathrm{Bohr}}\left(Z_{1}^{1 / 2}+Z_{2}^{1 / 2}\right)^{-2 / 3},
$$

which is expressed as a function of the atomic numbers $Z_{1}$ and $Z_{2}$ of the two atoms, and the Bohr distance $a_{\text {Bohr }}$ $=0.529 \AA$. The total potential energy can then be written as

$$
U_{\mathrm{tot}}(r)=f_{c}(r) U_{M}(r)+\left(1-f_{c}(r)\right) U(r),
$$

where $U(r)$ stands for the reactive many-body part of the potential described by Eq. (6). The switching range of the cutoff function $f_{c}(r)$ is the same for all atomic pairs, namely, $0.3 \AA<r<0.9 \AA$.

\section{THE FITTING PROCEDURE}

The calculations of energies, force constants, equilibrium bond distances, and angles in isolated molecules were performed with the GAMESS program. ${ }^{18}$ All calculations were done within DFT using B3LYP density functional and the
6-311G* basis set. The force constants were calculated from seminumerical Hessians in GAMESS. The properties of graphene and diamond were calculated with the ABINIT program ${ }^{19}$ within DFT, using the Perdew-Burke-Ernzerhof exchange-correlation generalized gradient approximation functional. ${ }^{20}$

Different bond types were labeled by listing the symbols of the elements forming the bond followed by the numbers of neighbors of each type on each side of the bond in parentheses. For instance, $\mathrm{CO}(2110)$ designates a carbon-oxygen bond with two carbons and one oxygen connected to a carbon atom and one carbon and no oxygens connected to an oxygen atom. When denoting bonds between identical atoms, the left and right parts in parentheses can be exchanged, e.g., both $\mathrm{CC}(1000)$ and $\mathrm{CC}(0010)$ refer to the same bond.

The angular spring constants in Eq. (13) were obtained from the calculated Hessians and equilibrium bond distances of linear triatomic molecules. The values of the coefficients in Eq. (13) for various combinations of atoms are given in Table II. The dihedral coefficient in Eq. (14) for C atoms 


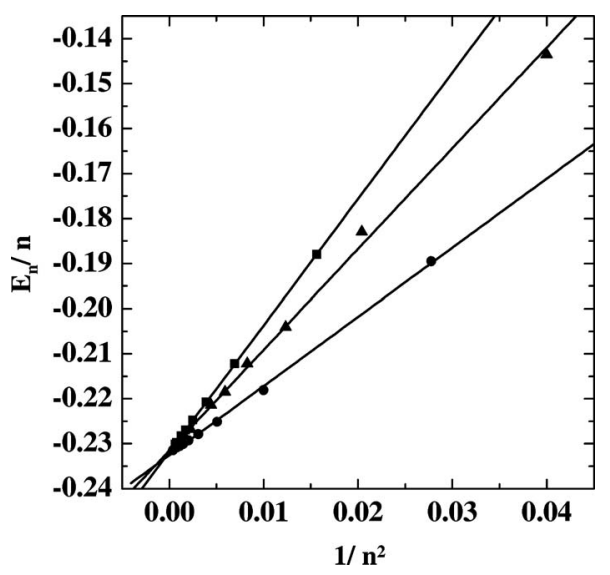

FIG. 2. Calculated energy of $n$-atom carbon rings $\mathrm{C}_{n}$ normalized by the number of atoms $n$ as a function of $1 / n^{2}$. (Circles) $n=4 k+2$; (squares) $n=4 k$; (triangles) odd $n$. The lines are linear fits to the calculated points.

$k_{\mathrm{CCCC}}^{\text {tor }}=1.583 \mathrm{eV}$ was deduced by comparing the energy of a graphene sheet with that of a hypothetical H-6 carbon structure, $^{21}$ in which atoms have the same threefold local coordination as in graphene, but in which one-third of dihedral angles are $60^{\circ}$ instead of $0^{\circ}$. All other dihedral interactions were not considered. The bond energies $U_{i j}$ are found by subtracting the angular and dihedral components from the total energy of the molecule. Since bonds of the same type can occur in more than one molecule, there is an ambiguity in the choice of molecules for the fitting. Here, molecules with the least number of atoms representing a given bond type were employed.

In symmetric bonding configurations, when all bonds are of the same type [e.g., a $\mathrm{CO}(0200)$ bond in a $D_{3 h} \mathrm{CO}_{3}$ ], the assignment of the energy to each of the bonds is obvious. In other molecules, such as a linear $\mathrm{C}-\mathrm{O}-\mathrm{O}$, where only a sum of the energies of the two bonds is known, the bond energies were defined to be proportional to the bond orders as calculated by GAMESS.

$\mathrm{CO}$ bond. The results of calculations for the $\mathrm{CO}$ bond are shown in Table III. Since the CO compounds are usually gases at normal conditions, their binding energy must quickly decrease with coordination. The calculated molecular energies listed in the table demonstrate that this is indeed the case. In diatomic carbon monoxide, carbon and oxygen form a strong covalent bond with the energy of $-11 \mathrm{eV}$. Connecting a second oxygen atom to the carbon atom results in a carbon dioxide molecule, predicted to be $5.7 \mathrm{eV}$ lower in energy than $\mathrm{CO}$. Being more stable than carbon monoxide, $\mathrm{CO}_{2}$ nevertheless has less energy per each of its two $\mathrm{CO}(0100)$ bonds. The molecular stability starts decreasing when more oxygen atoms are added on the $\mathrm{C}$ side of the $\mathrm{CO}$ bond. The calculated energy of $D_{3 h}$ carbon trioxide $\mathrm{CO}_{3}$ (Ref. 22) is slightly above $\mathrm{CO}_{2}$, and its bond energy is also smaller. The energy of $T_{d} \mathrm{CO}_{4}$ is above that of $\mathrm{CO}_{3}$ by $4.0 \mathrm{eV}$, although the formal bond strength is higher than in $\mathrm{CO}_{3}$ due to high contributions from the angular terms in this molecule. The molecular stability decreases even more rapidly when adding carbon atoms on the $\mathrm{O}$ side of the $\mathrm{CO}$ bond. The linear $\mathrm{C}_{2} \mathrm{O}$ molecule in its $X^{3} \Sigma^{-}$ground state ${ }^{23}$ is
TABLE IV. Coefficients for the linear fittings of the energies of $\mathrm{C}_{n}$ rings as described by Eq. (18).

\begin{tabular}{ccc}
\hline \hline & $U_{\mathrm{CC}(1010)}(\mathrm{eV})$ & $\mathrm{k}_{\mathrm{CCC}}(\mathrm{eV})$ \\
\hline $4 n$ & -6.308219187 & 3.872626914 \\
$4 n+2$ & -6.327616194 & 2.116749568 \\
Odd & -6.300275152 & 3.087086693 \\
\hline \hline
\end{tabular}

less stable than the carbon monoxide, while $D_{3 h} \mathrm{C}_{3} \mathrm{O}$ has the energy of only $-5.7 \mathrm{eV}$.

$O O$ bond. The calculations for the singlet ozone molecule predict an equilibrium $\mathrm{O}-\mathrm{O}-\mathrm{O}$ angle of $118.2^{\circ}$, and an atomization energy of $-5.81 \mathrm{eV}$, which is $0.54 \mathrm{eV}$ lower than the energy of the triplet ground state of $\mathrm{O}_{2}$. The parameters of the $\mathrm{OO}(0101)$ bond were determined from the calculation of the $D_{4 h}$ (square) $\mathrm{O}_{4}$ molecule, predicted to be less stable than $\mathrm{O}_{3}(-5.58 \mathrm{eV})$. Larger $\mathrm{O}$ atom clusters were not considered due to their instability. The energies of the $\mathrm{OO}$ bonds in configurations involving neighboring $\mathrm{C}$ atoms are listed in Table III.

$C C$ bond. Due to the greater electron delocalization in various spatially extended structures formed by carbon, local potentials may be expected to be less suitable for description of these structures than various $\mathrm{CO}$ molecules. While delocalization effects must be most pronounced in periodic solids exemplified by graphene or diamond, they are also found to be evident in small structures such as uniform rings of carbon atoms.

Carbon $\mathrm{C}_{n}$ rings and linear carbon chains. Uniform carbon rings $\mathrm{C}_{n}$ have $n$ equivalent $\mathrm{CC}(1010)$ bonds contributing equally to the total energy of the molecule. In order to maximize the scope of the fit, $\mathrm{C}_{n}$ rings of various sizes with $n$ between 5 and 50 were calculated. From Eq. (13) with $\theta_{0}=\pi$, it follows that

$$
E_{\text {total }}\left(\mathrm{C}_{n} \operatorname{ring}\right)=n U_{\mathrm{CC}(1010)}+\frac{2 k_{\mathrm{CCC}} \pi^{2}}{n} \text {. }
$$

According to Eq. (18), the plot of $E_{\text {total }}\left(\mathrm{C}_{n} \operatorname{ring}\right) / n$ vs $1 / n^{2}$ should be linear, yielding $k_{\mathrm{CCC}}$ and $U_{\mathrm{CC}(1010)}$ from the slope and intercept, correspondingly. Figure 2 shows this plot for $n=5-33$. The validity of the approximation that the total energy can be represented as a sum of the bond energy and angle energy can now be examined. Three distinct lines are generated, corresponding to $n=4 k+2, n=4 k$, and $n=2 k+1$. Within each of these three groups, it is permissible to describe the total energy with Eq. (18). The most stable molecules are aromatic rings with $n=4 k+2$, while the least stable are the rings with the even number of atoms not satisfying the aromaticity condition. The odd-membered rings have intermediate stability. The stability of ring molecules with $n=4 k+2$ originates from the formation of closed shell configurations, as described by Huckel's rule. The fitted values are given in Table IV, giving different types of molecules' different angular stiffness, while the bond energies are the same in all molecules. These calculations demonstrate the limitations of a local potential in approximating the energies of these molecules. Since the local potential cannot estimate the total number of atoms in the ring, a global quan- 


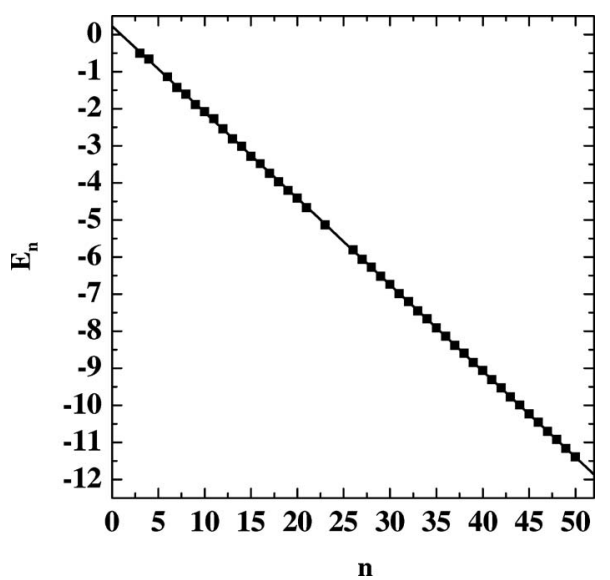

FIG. 3. Calculated energy of n-atom linear carbon molecules $\mathrm{C}_{n}$ as a function of $n$. The line gives a linear fit to the calculated points.

tity, it cannot account for the differences between the lines in Fig. 2. For the purposes of fitting the $\mathrm{CC}(1010)$ bond in the current potential, the values of $U_{\mathrm{CC}(1010)}$ and $k_{\mathrm{CCC}}$ were averaged over the corresponding values for $n=4 k+2, n=4 k$, and $n=2 k+1$ molecules.

Unlike homogeneous $\mathrm{C}_{n}$ rings, linear carbon molecules have $(n-3) \mathrm{CC}(1010)$ bonds, plus two terminating CC(1000) bonds. The energy of a linear carbon chain with $n$ atoms is

$$
E_{\text {total }}\left(\mathrm{C}_{n} \text { linear }\right)=(n-3) U_{\mathrm{CC}(1010)}+2 U_{\mathrm{CC}(1000)} \text {. }
$$

According to Eq. (19), $E_{\text {total }}\left(\mathrm{C}_{n}\right.$ linear $)$ yields a line when plotted as a function of $n$. Figure 3 shows the calculated $E_{\text {total }}\left(\mathrm{C}_{n}\right.$ linear $)$ as a function of $n$ for $n=3-50$, as well as a linear fit, yielding $U_{\mathrm{CC}(1010)}=-6.327 \mathrm{eV}$ and $U_{\mathrm{CC}(1000)}$ $=-0.6403 \mathrm{eV}$. As expected, the obtained value of $U_{\mathrm{CC}(1010)}$ is very close to the value $U_{\mathrm{CC}(1010)}=-6.321 \mathrm{eV}$ obtained from the fitting of $\mathrm{C}_{n}$ rings.

Graphene structures. The energy and equilibrium distance of the $\mathrm{CC}(2020)$ bond in a single graphene sheet obtained with ABINIT simulations are given in Table III. The force constant of $45.0 \mathrm{eV} / \AA^{2}$ based on the experimentally measured elastic stiffness coefficients $\mathrm{C}_{11}$ and $\mathrm{C}_{12}$ of graphite $^{24}$ was used for this bond.

The energies of defects in graphene were calculated with GAMESS using a $\mathrm{C}_{56} \mathrm{H}_{18}$ molecule shown in Fig. 4. After optimizing the geometry of the molecule, atoms 1-6 were removed and the energy of the resulting structures was calculated. Removal of atoms 1-6 is equivalent to the elimination of $12 \mathrm{CC}(2020)$ bonds, and conversion of another 12 CC(2020) bonds into CC(1020). The obtained energy of the $\mathrm{CC}(1020)$ bond is given in Table III. The parameters of the $\mathrm{CC}(3030)$ bond were obtained from the calculation of the equilibrium structure of diamond. Other carbon-carbon bonds are also given in Table III.

\section{MD SIMULATION OF GRAPHENE UNZIPPING BY OXYGEN}

As a test of our potential, a classical molecular dynamics (MD) simulation was performed to describe the ordered oxidation and subsequent unzipping and cracking of graphene sheets by chemisorbed oxygen. Using ab initio DFT calcula-

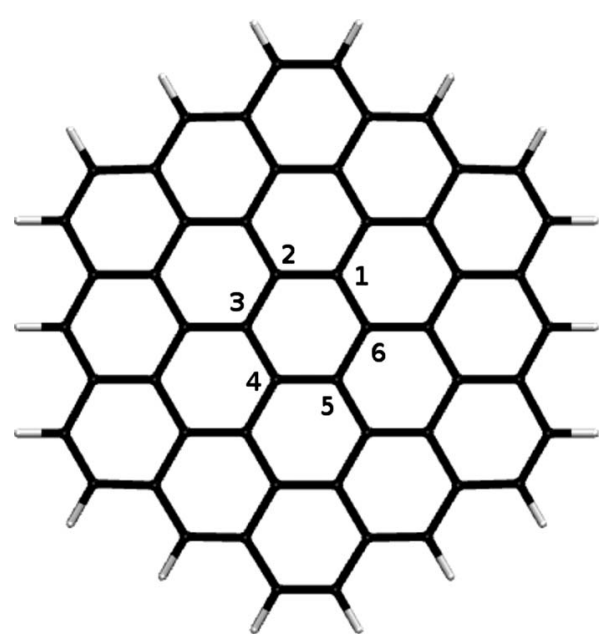

FIG. 4. A GAMESS-optimized structure of the $\mathrm{C}_{56} \mathrm{H}_{18}$ molecule. Numbers 1-6 mark the atoms that were removed in order to evaluate the energy of the $\mathrm{CC}(1020)$ bond. The illustrations of molecules have been prepared using the package VMD (Ref. 26).

tions, Li et al. ${ }^{9}$ first described the reaction mechanism that causes oxygen-driven unzipping of graphitic materials. They found that cooperative unzipping ensues as a result of the formation of linear arrays of epoxy groups on opposite sites of hexagons in graphene. In our simulation, we adopted the same setup: oxygen atoms were placed above $\mathrm{C}-\mathrm{C}$ bonds in graphene along the opposite sides of hexagons, as shown in Fig. 5(a). Periodic boundary conditions were assumed. The system was attached to a $1200 \mathrm{~K}$ thermostat and then allowed to evolve according to Newtonian mechanics. Figures 5(b)-5(d) depict snapshots of the unzipping process of graphene by adsorbed oxygen atoms. The first $\mathrm{C}-\mathrm{C}$ bond is cleaved by an $\mathrm{O}$ atom at $t=349 \mathrm{ps}$, creating a defect site. Then, unzipping is initiated at this site and proceeds serially in both directions along the linear array of adjacent oxygen atoms. Figure 5(c) captures the breakage of the second carbon-carbon bond next to the first epoxy group, which occurs at $t=459 \mathrm{ps}$. Eight $\mathrm{C}-\mathrm{C}$ bonds unravel after $1061 \mathrm{ps}$, as shown in Fig. 5(d). All $\mathrm{C}-\mathrm{C}$ bonds become disconnected at $t=1163 \mathrm{ps}$. All bond cleavages subsequent to the first one occur in a chain fashion next to the already cleaved bonds, taking on average 90 ps per each $\mathrm{C}-\mathrm{C}$ bond. This unzipping process is irreversible: No restoration of the $\mathrm{CC}$ bonds has been seen in the simulation up to $t=1800 \mathrm{ps}$. After cleaving a $\mathrm{C}-\mathrm{C}$ bond, each oxygen atom remains connected to two carbons, oscillating between two buckled positions on either side of the graphene plane. Our results agree well with the observations and predictions made by $\mathrm{Li}$ et al. ${ }^{9}$ for a

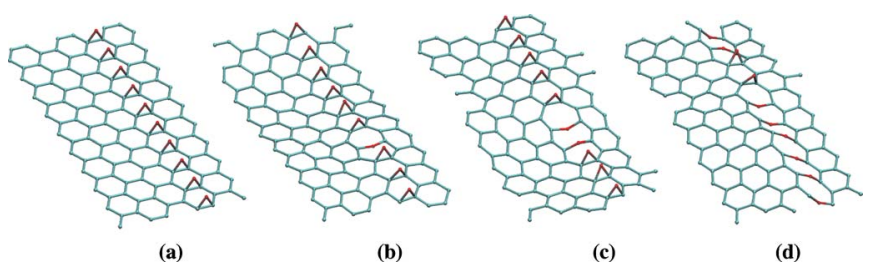

FIG. 5. (Color online) Snapshots from the simulation of graphene unzipping by oxygen atoms at $T=1200 \mathrm{~K}$. The snapshots are taken at (a) $t=0 \mathrm{ps}$, (b) $t=349 \mathrm{ps}$, (c) $t=459 \mathrm{ps}$, and (d) $t=1061 \mathrm{ps}$. 


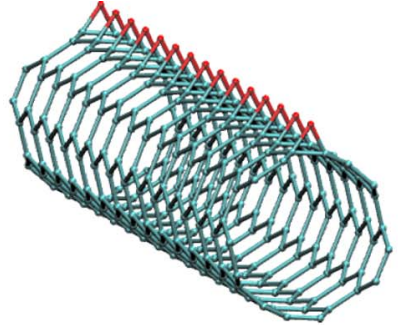

(a)

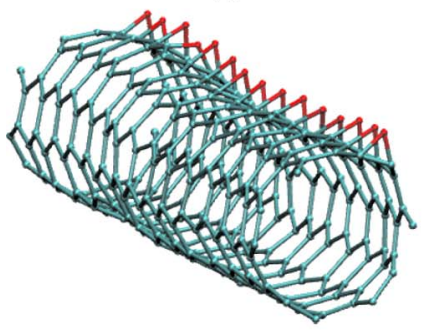

(b)

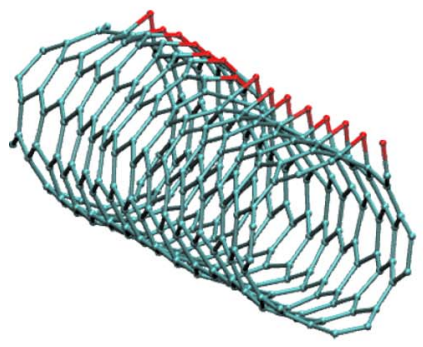

(c)

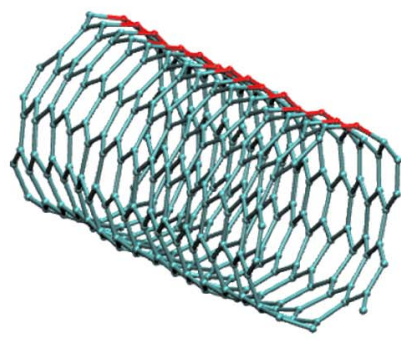

(d)

FIG. 6. (Color online) Snapshots from the simulation of unzipping of a $(5,5)$ nanotube by oxygen at $T=1200 \mathrm{~K}$. (a) The initial configuration at time zero showing the oxygen atom placement. Snapshots of the system after (b) 114 (c) 180, and (d) 296 ps of simulation time.

smaller graphene system (coronene molecule), based on first principles quantum mechanical calculations.

Li et al. also suggested that the unzipping mechanism may be operative in the breaking of oxidized carbon nanotubes. They speculated that serial breaking of $\mathrm{C}-\mathrm{C}$ bonds in the circumferential direction could explain the controllable shortening of carbon nanotubes by chemical oxidation. ${ }^{25}$ The unzipping of single-walled nanotubes (SWNTs) by oxidation offers a good system to further implement our potential. We focus here on the unzipping of SWNTs in the direction parallel to the nanotube axis.

The oxidative unzipping of a $(5,5)$ SWNT was modeled in order to investigate the role of the wall curvature on the unzipping process. In the initial configuration [Fig. 6(a)], oxygen atoms were placed along the axial direction on the

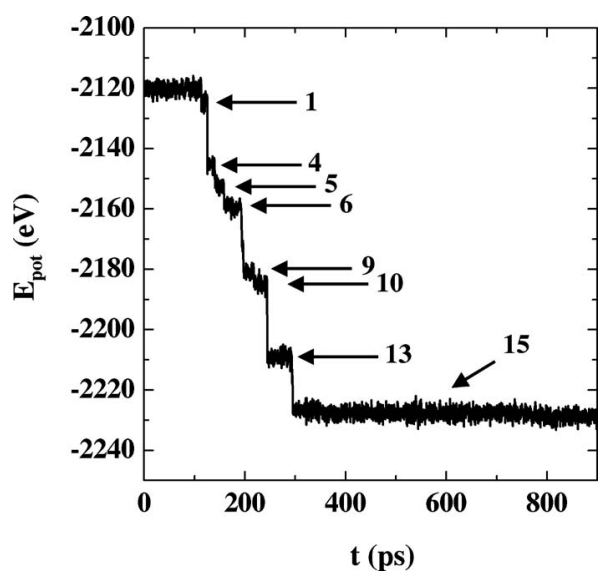

FIG. 7. Total potential energy for a system consisting of a $(5,5)$ nanotube segment, externally decorated with 15 oxygen atoms, as a function of time during the unzipping process. The arrows indicate the point at which a particular oxygen-decorated $\mathrm{C}-\mathrm{C}$ bond breaks in succession.

outer surface of the nanotube. As in the previous case of the flat graphene sheet, the oxygen atoms were positioned above the opposite sides of hexagons forming a row. Figure 6(b) shows the system at $1200 \mathrm{~K}$ after $114 \mathrm{ps,}$, when the first $\mathrm{C}-\mathrm{C}$ bond is cleaved. Note that bond splitting by an $\mathrm{O}$ atom occurs much earlier than in the case of graphene. Once the first $\mathrm{C}-\mathrm{C}$ bond on the nanotube is split, adjacent bond splitting follows at an accelerated rate. Indeed, six $\mathrm{C}-\mathrm{C}$ bonds have been split at $180 \mathrm{ps}$, as shown in Fig. 6(c), whence it takes a lot longer to split just the second $\mathrm{C}-\mathrm{C}$ bond in flat graphene. We believe that the stress caused by the curvature of the nanotube wall is responsible for the faster unzipping. All $\mathrm{C}-\mathrm{C}$ bonds under the $\mathrm{O}$-atom row have been cleaved after $296 \mathrm{ps}$ of system evolution, as shown in Fig. 6(d). In this configuration, the cross section of the nanotube becomes oval. This deformation is attributed to the relaxation of the stressed wall.

Bond breaking releases energy, which can be calculated. Figure 7 depicts the total system (nanotube plus oxygen atoms) energy as a function of time during the unzipping process. It is clear from these figures that bond breaking occurs in steps. After the first $\mathrm{C}-\mathrm{C}$ bond breaks (a random event), it appears that adjacent bonds may break individually or in a cascade. Several picoseconds after the first $\mathrm{C}-\mathrm{C}$ bond breaks, three bonds break in rapid succession, appearing as a steep drop. Similar concerted bond breaking in rapid succession occurs after the 6th, 10th, and $13 \mathrm{~h}$ bonds are broken. As Li et al. suggested, energy released from the breaking of the first $\mathrm{C}-\mathrm{C}$ bond can induce cleavage of adjacent bonds. It is plausible that vibrations may accelerate or delay the cleavage of the next bond depending on which way the carbon atoms vibrate relative to each other. Thus, we suspect that a cascade may occur when the atoms of multiple bonds in adjacent hexagons move away from each other simultaneously.

\section{CONCLUSIONS}

We presented a reactive potential with environmentdependent bond strengths describing interactions between carbon and oxygen atoms. Unlike previous reactive potentials, the requirement to follow the Pauling rule was relaxed, 
providing more flexibility in reproducing binding energies in molecules. The potential was tested in a simulation of oxidative unzipping of graphene sheets and carbon nanotubes. Oxygen atoms located on the opposite sides of hexagons in graphene split the carbon-carbon bonds beneath them. The unzipping along a chain of oxygen atoms is facilitated by the creation of an original defect site. Carbon nanotubes, with oxygen atoms placed on its outer surface, unzip much faster than flat graphene sheets.

\section{ACKNOWLEDGMENTS}

This material was based on work partially support by NSF Grant No. CTS-0613981.

${ }^{1}$ J. Tersoff, Phys. Rev. B 37, 6991 (1988).

${ }^{2}$ D. W. Brenner, Phys. Rev. B 42, 9458 (1990).

${ }^{3}$ D. W. Brenner, O. A. Shenderova, J. A. Harrison, S. J. Stuart, B. Ni, and S. B. Sinnott, J. Phys.: Condens. Matter 14, 783 (2002).

${ }^{4}$ A. C. T. van Duin, S. Dasgupta, F. Lorant, and W. A. Goddard, J. Phys. Chem. A 105, 9396 (2001).

${ }^{5}$ C. F. Abrams and D. B. Graves, J. Appl. Phys. 86, 5938 (1999); D. Humbird and D. B. Graves, J. Chem. Phys. 120, 2405 (2004).

${ }^{6}$ B. Ni, K.-H. Lee, and S. B. Sinnott, J. Phys.: Condens. Matter 16, 7261 (2004).

${ }^{7}$ I. Jang and S. B. Sinnott, J. Phys. Chem. B 108, 18993 (2004).

${ }^{8}$ H. Lodish, Molecular Cell Biology (Freeman, San Francisco, CA, 2003).

${ }^{9}$ J.-L. Li, K. N. Kudin, M. J. McAllister, R. K. Prud'homme, I. A. Aksay, and R. Car, Phys. Rev. Lett. 96, 176101 (2006).
${ }^{10}$ D. Troya, R. Z. Pascual, D. J. Garton, T. K. Minton, and G. C. Schatz, J. Phys. Chem. A 107, 7161 (2003).

${ }^{11}$ R. D. Bath, J. L. Andres, M.-D. Su, and J. J. W. McDouall, J. Am. Chem. Soc. 115, 5768 (1993).

${ }^{12}$ S.-H. Jhi, S. G. Louie, and M. L. Cohen, Phys. Rev. Lett. 85, 1710 (2000).

${ }^{13}$ G. C. Abell, Phys. Rev. B 31, 6184 (1985).

${ }^{14}$ J. H. Rose, J. R. Smith, F. Guinea, and J. Ferrante, Phys. Rev. B 29, 2963 (1984).

${ }^{15}$ L. Pauling, The Nature of the Chemical Bond (Cornell University Press, Ithaca, NY, 1960)

${ }^{16}$ G. Moliere, Z. Naturforsch. A 2A, 133 (1947).

${ }^{17}$ O. B. Firsov, Sov. Phys. JETP 5, 1192 (1957).

${ }^{18}$ M. W. Schmidt, K. K. Baldridge, J. A. Boatz, S. T. Elbert, M. S. Gordon, J. H. Jensen, S. Koseki, M. Natsunaga, K. A. Nguyen, S. J. Su, T. L. Windus, M. Dupuis, and J. A. Montgomery, J. Comput. Chem. 14, 1347 (1993).

${ }^{19}$ X. Gonze, J.-M. Beuken, R. Caracas, et al., Comput. Mater. Sci. 25, 478 (2002).

${ }^{20}$ J. P. Perdew, K. Burke, and M. Ernzerhof, Phys. Rev. Lett. 77, 3865 (1996).

${ }^{21}$ M. A. Tamor and K. C. Hass, J. Mater. Res. 5, 2273 (1990).

${ }^{22}$ C. S. Jamieson, A. M. Mebel, and R. I. Kaiser, ChemPhysChem 7, 2508 (2006).

${ }^{23}$ C. F. Chabalowski, S. D. Peyerimhoff, and R. J. Buenker, J. Chem. Phys. 84, 268 (1986)

${ }^{24}$ O. L. Blakslee, D. G. Proctor, E. J. Seldin, G. B. Spence, and T. Weng, J. Appl. Phys. 41, 3373 (1970).

${ }^{25}$ J. Liu, A. G. Rinzler, H. Dai, J. H. Hafner, R. K. Bradley, P. J. Boul, A. Lu, T. Iverson, K. Shelimov, C. B. Huffman, F. Rodriguez-Macias, Y.-S. Shon, T. R. Lee, D. T. Colbert, and R. E. Smalley, Science 280, 1253 (1998).

${ }^{26}$ W. Humphrey, A. Dalke, and K. Schulten, J. Mol. Graphics 14, 33 (1996). 